\title{
Calibrated X-ray micro-tomography for mineral ore quantification
}

\author{
F. Reyes ${ }^{1}$, Q. Lin ${ }^{1}$, O. Udoudo ${ }^{2}$, C. Dodds ${ }^{2}$, P.D. Lee ${ }^{3}$, S. Neethling ${ }^{1 *}$ \\ 1 Department of Earth Science and Engineering, Imperial College London, London SW7 2AZ, UK \\ 2 Microwave Process Engineering Research Group, Faculty of Engineering, University of Nottingham, Nottingham NG7 \\ $2 R D, U K$ \\ 3 School of Materials, University of Manchester, Manchester M13 9PL, UK
}

Keywords: X-ray micro-CT, Mineral Liberation Analysis, Mineral characterisation

\begin{abstract}
Scanning Electron Microscopy (SEM) based assessments are the most widely used and trusted imaging technique for mineral ore quantification. X-ray micro tomography (XMT) is a more recent to the mineralogy toolbox, but with the potential to extend the measurement capabilities into the three dimensional (3D) assessment of properties such as mineral liberation, grain size and textural characteristics. In addition, unlike SEM based assessments which require the samples to be sectioned, XMT is non-invasive and non-destructive manner. The disadvantage of XMT, though is that the mineralogy must be inferred from the X-Ray attenuation measurements, which can make it hard to distinguish from one another, whereas SEM when coupled with Energy-Dispersive X-ray Spectroscopy (EDX) provides elemental compositions and thus a more direct method for distinguishing different minerals. A new methodology that combines both methods at the mineral grain level is presented. The rock particles used to test the method were initially imaged in 3D using XMT followed by sectioning and the 2D imaging of the slices using SEM-EDX. An algorithm was developed that allowed the mineral grains in the 2D slice to be matched with their 3D equivalents in the XMT based images. As the mineralogy of the grains from the SEM images can be matched to a range of X-Ray attenuations, this allows minerals which have similar attenuations to one another to be distinguished, with the level of uncertainty in the classification quantified. In addition, the methodology allowed for the estimation of the level of uncertainty in the quantification of grain size by XMT, the assessment of stereological effects in SEM 2D images and ultimately to obtain a simplified 3D mineral map from low energy XMT images. Copper sulphide ore fragments, with chalcopyrite and pyrite as the main sulphide minerals, were used to demonstrate the effectiveness of this procedure.
\end{abstract}




\section{Introduction}

Quantification by imaging techniques has given valuable insights into the internal structure and textural characteristics of mineral samples. Many of these assessments have become indispensable for relating the performance of a variety of mineral processes with mineralogy quantification (Kyle \& Ketcham, 2015). Applications include liberation processes such as milling (Leißner et al., 2016), recovery processes such as flotation (Jordens et al., 2016, Tungpalan et al., 2015) and heap leaching (Naderi et al., 2011). Two of the main analytical devices used for imaging the internal structure of mineral ores are X-ray micro tomography (XMT) and Scanning Electron Microscopy (SEM) based mineral analysis (Sutherland \& Gottlieb, 1991; Cnudde \& Boone, 2013; Lin et al. 2016b).

XMT is a non-invasive and non-destructive tool that has been used for assessing ore samples in terms of, for instance, their mineral liberation, fracture network, gangue porosity and grain size distributions (Garcia et al., 2009; Ghorbani et al., 2011; Dhawan et al., 2012; Charikinya et al., 2015). For example, Miller et al., (2003) studied the relationship between ultimate recovery in heap leaching and mineral exposure, building a predictive model based on the exposure measured from XMT images. Despite being a versatile tool, XMT images do not directly provide the mineralogy of the sample under study, which is a major drawback for geological and mineral processing applications.

Scanning Electron Microscopy (SEM) based mineral analysis can be used to obtain mineralogical information of the ore. Equipment based on a combination of SEM and Energy-Dispersive X-ray Spectroscopy (EDS or EDX) measurements, implemented commercially as MLA (Mineral Liberation Analyser) or QEMSCAN (Quantitative Evaluation of Minerals by Scanning Electron Microscopy) are widely used for the majority of mineral assessments (Fandrich et al., 2007) ${ }^{1}$. Other commercial devices include Zeiss Mineralogic, TESCAN TIMA and Oxford Inca. These techniques require sectioning of the fragments to expose $2 \mathrm{D}$ surfaces to reveal the internal structure. In contrast to XMT this technique is thus destructive and invasive, but presents better image resolution and classifies the different minerals present in the sample by a composite of electron microscopy and Xray data. Another drawback of SEM-based analysis is that only 2D information is accessible, making stereological corrections advisable when size and mineral liberation quantification are performed. SEM and XMT have been used together in order to take advantage of their complementary assessment. Gay \& Morrison (2006) developed a correction methodology to infer 3D information

\footnotetext{
${ }^{1}$ In this paper 'SEM/EDS' is used to refer to any machine with both capabilities, like MLA or QEMSCAN. Whilst, 'SEM' is used to refer to the analysis obtained from those machines, including the SEM and EDS information.
} 
from 1D and 2D information given by 2D SEM images. The methodology was then corroborated with 3D XMT images of the same sectioned fragments. However, only the cumulative liberation curves were used for comparison between both techniques, with no grain-by-grain analysis carried out. The validation was thus indirect, with no measurement of the relationship between the actual grain size and the distribution of sizes seen in the section and how this stereology error varies with the grain size. Sok et al. (2010) used high resolution SEM images to correlate local pore size and microporosity with the X-ray attenuation obtained from low resolution scans. Adding this calibrated micro-porosity to the macro-porosity readily measured in XMT allowed good agreement to be achieved with mercury porosimetry data. Van Geet et al. (2001), on the other hand, used SEM and optical imaging to identify mineralogical phases in coal samples. In none of the presented works were the levels of confidence and uncertainty bounds assessed.

In this paper we propose a novel methodology for quantifying mineral information by combining information from XMT and SEM-based assessments using a grain-by-grain approach. We demonstrate how SEM-based mineral information can be used to calibrate XMT image segmentation to provide quantitative 3D mineralogical data. In addition, the XMT 3D information is used to assess stereological effects in SEM images, providing a method for easily generating ore and mineralogy specific stereological corrections. The novelty of the proposed methodology is that it allows the confidence bounds in the mineral segmentation and classification to be assessed, important considerations if these methods are to be used for quantitative analysis. The methodology is tested on copper sulphide ore fragments containing mainly chalcopyrite and pyrite in a quartz gangue matrix. The reason for choosing this ore is because chalcopyrite and pyrite are commonly associated with one another and yet are notoriously difficult to distinguish from one another in XMT images.

\section{Materials and methods}

\subsection{Ore samples}

For this paper a typical porphyry copper ore with a grade of $0.8 \mathrm{wt} \%$ copper was used. Table 1 summarises the mineral composition as obtained from SEM assessment. The main non-sulphide gangue mineral is quartz, with pyrite being the main sulphide constituent, though it is considered a gangue mineral as the copper containing minerals are the species of interest. The main copper mineral is chalcopyrite, though accompanied by a range of other copper containing species. The fragments consisted of crushed ore in the 8 to $12.22 \mathrm{~mm}$ size range. 
Table 1. Main mineral composition of the ore sample. Primary and secondary sulphides along with predominantly quartz gangue are the main minerals present in the ore samples used. The variability is the fragment to fragment standard deviation in the measurements.

\begin{tabular}{lccc}
\hline Mineral type & Weight \% & Specific gravity & ID \\
\hline $\begin{array}{l}\text { Copper containing } \\
\text { species }\end{array}$ & $\mathbf{2 . 1 3 \pm 0 . 4 7}$ & & \\
$\begin{array}{l}\text { Bornite } \\
\text { Chalcopyrite }\end{array}$ & 0.25 & 5.06 & $\mathrm{Cu}_{5} \mathrm{FeS}_{4}$ \\
Chalcocite & 1.25 & 4.1 & $\mathrm{CuFeS}_{2}$ \\
Covellite & 0.011 & 5.5 & $\mathrm{Cu}_{2} \mathrm{~S}$ \\
Tennantite & 0.136 & 4.6 & $\mathrm{CuS}$ \\
& 0.48 & 4.65 & $\mathrm{Cu}_{6}\left[\mathrm{Cu}_{4}\left(\mathrm{Fe}_{2} \mathrm{Zn}\right)_{2}\right] \mathrm{As}_{4} \mathrm{~S}_{13}$ \\
Pyrite & $\mathbf{7 . 0 0 \pm 2 . 7 4}$ & 4.95 & $\mathrm{FeS}_{2}$ \\
Gangue minerals & & & \\
Muscovite & $\mathbf{9 0 . 6 9 \pm 2 . 7 3}$ & & $\mathrm{KAl}_{2}\left(\mathrm{AlSi}_{3} \mathrm{O}_{10}\right)\left(\mathrm{F}, \mathrm{OH}_{2}\right.$ \\
Quartz & 39.81 & 2.76 & $\mathrm{SiO}_{2}$ \\
Other minerals & 41.93 & 2.65 &
\end{tabular}

\subsection{XMT scanner}

A Nikon XTH 225 scanner was used to carry out the XMT acquisition, 37 fragments were analysed in total with 34 of them XMT scanned at high energy levels and lower resolution. The remaining three were XMT scanned at higher resolution and lower energy levels. The detector consists of a matrix of $2000 \times 2000$ detectors, which gave a linear resolution of approximately 9 to $16 \mu \mathrm{m}$ for the size range of fragments used. The parameters used for the acquisition are listed in Table 2.

Table 2. Scanner conditions for XMT scans.

\begin{tabular}{lcc}
\hline & \multicolumn{2}{c}{ Value } \\
\cline { 2 - 3 } Parameter & Low Energy & High Energy \\
\hline Voltage & $50 \mathrm{kV}$ & $89 \mathrm{kV}$ \\
Current & $137 \mu \mathrm{A}$ & $117 \mu \mathrm{A}$ \\
Filter & none & $1 \mathrm{~mm} \mathrm{Al}$ \\
Exposure & $2 \mathrm{~s}$ & $0.708 \mathrm{~s}$ \\
Projections & 2001 & 2001 \\
Resolution & $8.9 \mu \mathrm{m}$ & $16.1 \mu \mathrm{m}$
\end{tabular}

Using the database created by Berger et al. (2010), the linear attenuation coefficient can be plotted for different X-ray energy levels. Figure 1 shows this coefficient for the main minerals encountered in the samples studied in this paper. There is a significant gap in attenuation between the quartz 
and the sulphide minerals. Note that although quartz was the main gangue mineral in these samples, the other non-sulphide gangue species also have much lower X-ray attenuations than the sulphide species. Segmentation difficulties arise when attempting to distinguish between the pyrite and chalcopyrite as their X-ray attenuation values are very similar to one another. It can be observed, though, that at lower energy levels the gap in the attenuation between these minerals is larger.

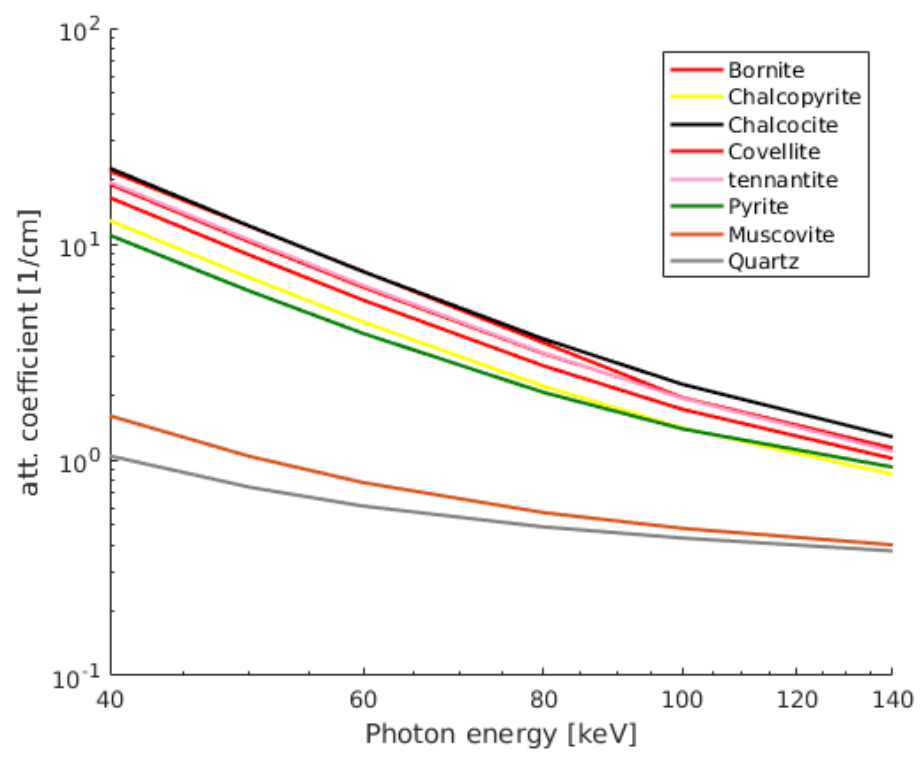

Figure 1 Linear attenuation of X-rays at different energy levels. Linear attenuation coefficient dependant on material density and effective atomic number varies with the energy level applied, phenomena exploit by dual energy radiography. (Berger et al., 2010)

However, the fraction of photons that are able to pass through the sample decreases with energy. As the signal-to-noise ratio depends on the number of photons detected, lower energy scans need longer exposure times. For the ore used, good contrast and a reasonable acquisition time was achieved using an energy level of $50 \mathrm{kV}$ at $137 \mu \mathrm{A}$ with a 2 second exposure time.

\subsection{SEM/EDS analysis}

After XMT scanning, all the fragments were ground and polished to expose a section of their interior for analysis within the FEI Quanta 600i SEM/MLA machine. The sample preparation involved mounting the fragments in $30 \mathrm{~mm}$ blocks using epoxy resin. The prepared sample blocks were then polished using the Struers Tegrapol polishing machine to a diamond finish of $1 \mu \mathrm{m}$. Carbon coating was also performed using the Quorum Q150R carbon coater to prevent electron charging of the exposed surface, avoiding image artefacts created by electrons being accumulated in sections of the ore. Figure 2 shows a schematic of the slice exposed for an example fragment; depending on the surface of the fragments, a slice 150 to $250 \mu \mathrm{m}$ deep was exposed. 


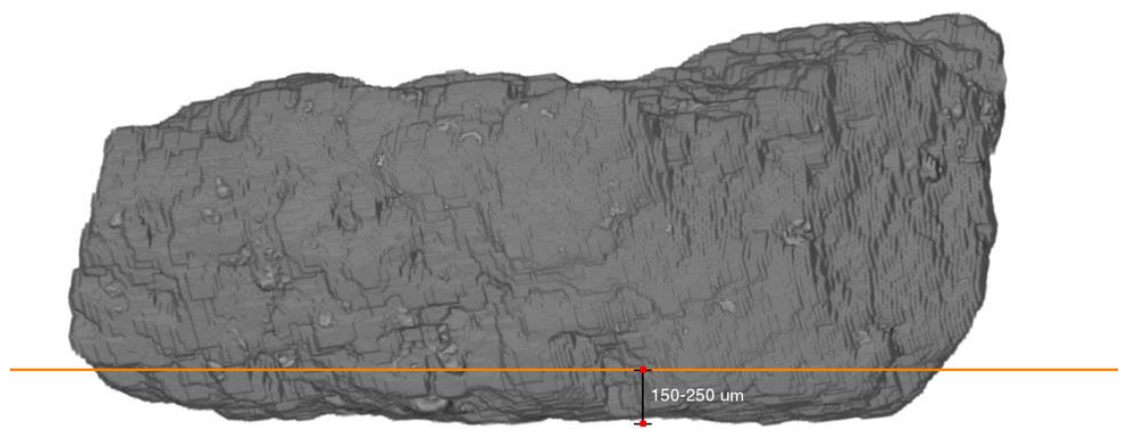

Figure 2 Example schematic of fragment's polished sectioning. Fragments were polished to different depths as shown in the figure by the orange slice.

A combination of a standard Back Scattered Electron (BSE) and a Grain-based X-ray Mapping (GXMAP) measurement methods (Fandrich et al., 2007) were performed in the SEM/MLA, at a resolution of $2.25 \mu \mathrm{m}$ per pixel. Figure 3 shows a region of interest in which the results from the 3 imaging modalities are compared. From left to right these are the low energy XMT image, the BSE image from the SEM, and a mineral map based on a combination of the SEM and EDS measurements. It should be noted that the XMT image represents a virtual 2D slice through a 3D data set (2D-XMT), while the other two images are inherently 2D data. It can be seen that the SEM BSE image has better contrast and looks sharper than the XMT image. Nevertheless, the difference between pyrite (green in the mineral map) and copper minerals (pink and red) is apparent in the $\mathrm{XMT}$ image when a low energy level is used.

a)

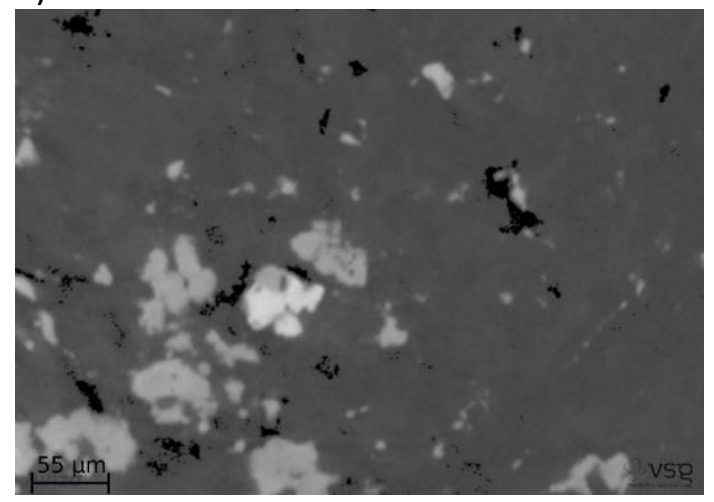

b)

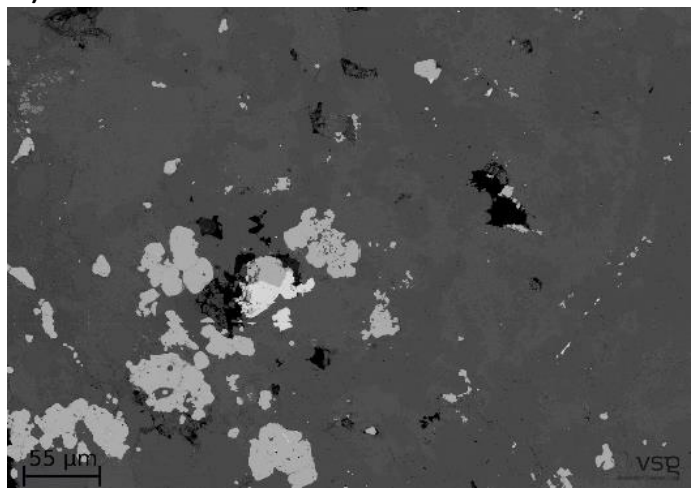

c)

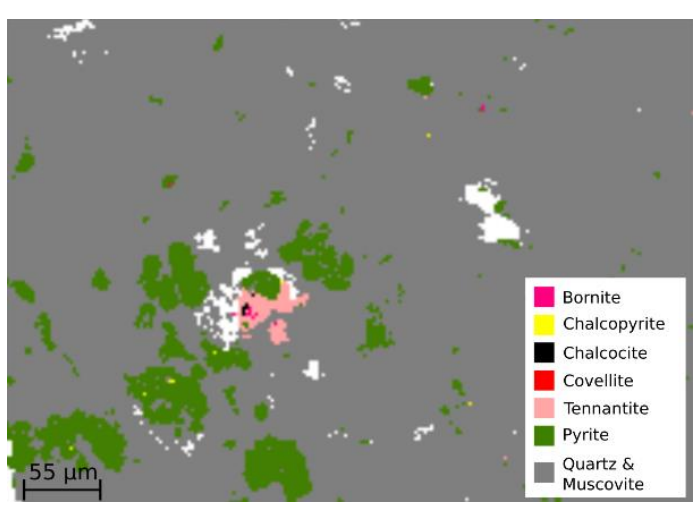

Figure 3 Region of interest of one example fragment. Contrast between gangue, pyrite and copper minerals is apparent in both modalities. a) low energy 2D-XMT image, b) BSE image and c) Mineral map showing classification given by the SEM analyser. 


\subsection{Grain level calibration of XMT scans using SEM-based analysis}

For the low energy XMT scans the pixel intensity histogram, depicted in Figure 4 for a particular fragment, shows clear peaks for some of the phases of interest. The two main peaks are due to gangue and the air and porous media in the particle. At higher intensities there are slight undulations in the histogram due to the presence of pyrite and the copper minerals, though these are not nearly as distinct as the other peaks. The methodology of using the SEM-based data to set the thresholds between these phases, as well as quantifying the resultant uncertainty in the classification, is the main focus of this paper.

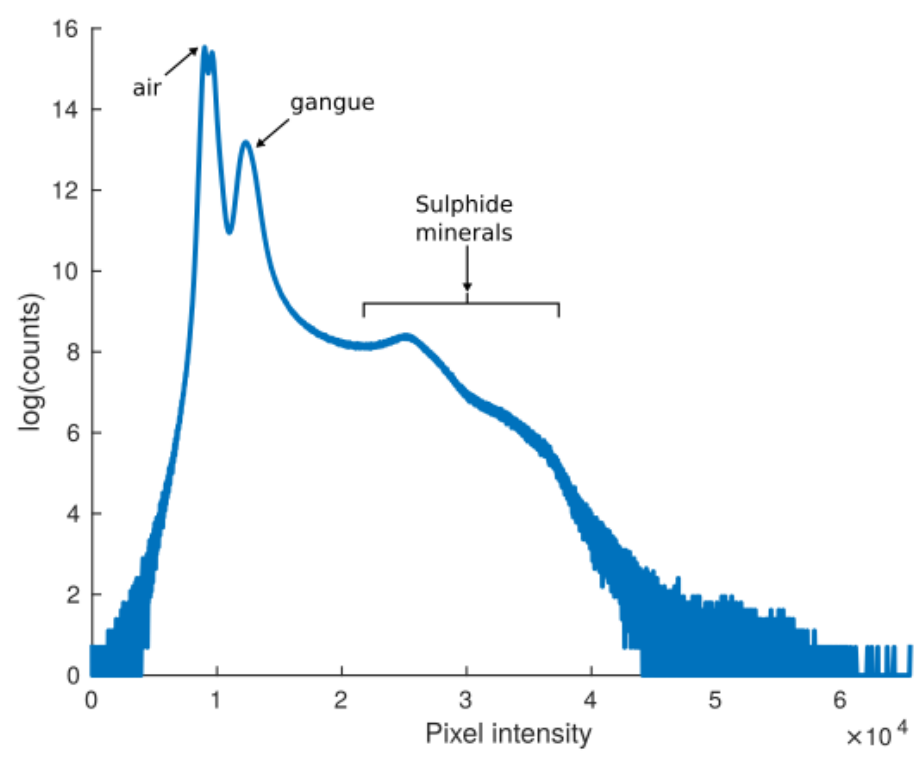

Figure 4 Example histogram of one XMT scan. Peaks from the key materials present in the sample are apparent, including air, gangue and sulphide minerals.

The algorithm proposed is based on the one developed by Lin et al. (2015 \& 2016a) and extended significantly to incorporate the SEM-based calibration. The methodology for combining XMT images and SEM sectioning, performed by a combination of routines in both Avizo ${ }^{\circledR}$ and Matlab ${ }^{\circledR}$, is as follows:

1. Fragments are individually scanned in the X-ray Computed Tomography scanner using the settings reported in Table 2.

2. Gangue matrix and bright phase are together segmented from air, using the Otsu algorithm (Otsu, 1979), setting the image intensity for the surrounding air and internal porosity to zero.

3. The bright phases (pyrite and copper minerals together) are segmented from the gangue using the maximum entropy global thresholding algorithm for each fragment (Kapur et al., 1985). The binary mask obtained is then labelled using a connectivity algorithm, with an 8connected neighbourhood, to identify individual grains.

4. Fragments are scanned in the SEM machine after a grinding and polishing procedure. 
5. Porosity is segmented in the BSE image of the sections in the same manner as described in step 2.

6. Minerals of interest are segmented from the mineral map image, in order to produce a binary mask. This is subsequently labelled as in step 3 .

7. The XMT 3D volume is then registered into the segmented BSE image so that a rigid motion transformation is obtained.

8. The motion transformation is used to align the XMT grain labelled mask (step 3 ) into the grain labelled mask (step 6), so that individually identified grains in both images have the same label.

A point to be stressed is that only the labelled images are transformed and matched, the original images (3D XMT, BSE and GXMAP measurements) are kept intact to avoid introducing systematic errors due to the registration process. This, however, is not the case for the virtual 2D XMT image, where the slice has to be created from the XMT 3D volume using registration algorithms. Figure 5 shows the BSE image of an example fragment slice along with the mineral map provided by GXMAP and the registered XMT grain labelled mask over the same BSE image. A good correspondence between all images can be observed.

a)

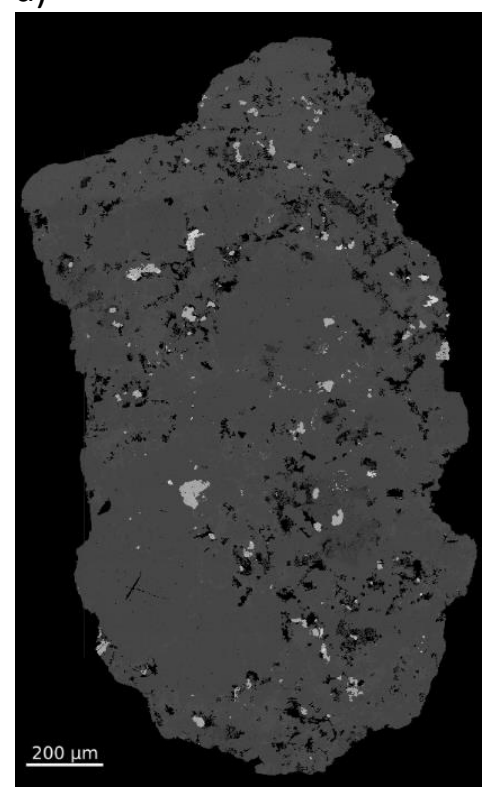

b)

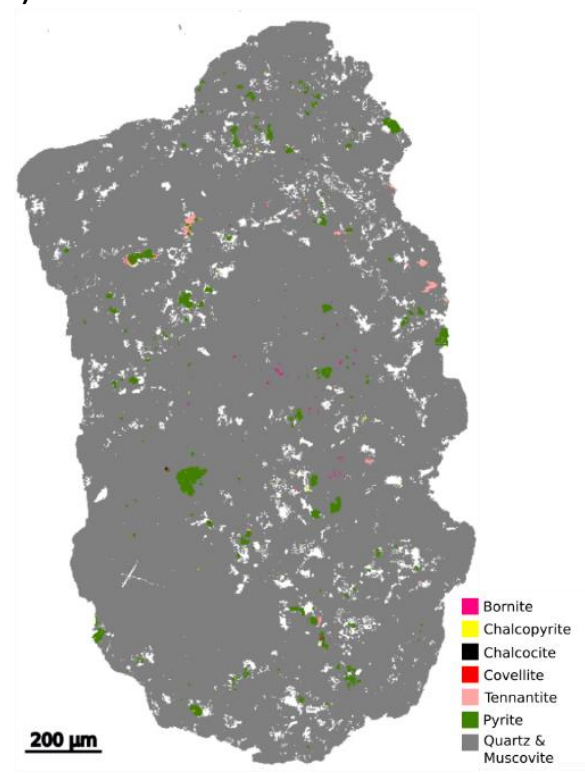

c)

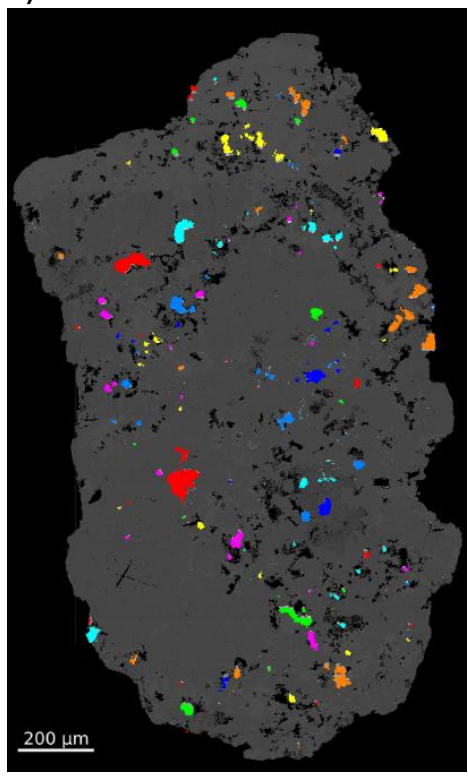

Figure 5 Registered slices from SEM and XMT analysis. The proposed grain tracking algorithm was applied to the SEM and XMT images to correlate information from each device. a) shows the BSE image from SEM, b) shows the mineral map from SEM and c) shows the XMT mineral label image overlapped to the BSE image, colours indicate the unique label number of each mineral grains.

After the algorithm has been applied to the ore samples, a grain-level database is created so that information about their pixel intensity, size and shape in XMT and BSE images, distance to the surface and mineralogy is available. This database is used to study variability in grain size quantification by XMT, stereological effects in grain size distribution obtained from SEM images and 
to calibrate a global threshold to segment the bright phase in XMT images so that pyrite and coppermineral grains can be distinguished from one another. Figure 6a shows the pixel intensity histogram as obtained from the XMT, showing no obvious value for a threshold. It should be noted that unlike Figure 4 this histogram is only for the pixels in the XMT 2D slices. An appropriate threshold can be found by plotting all mineral grains according to their mineralogy as given by the EDS-based classification against their image intensity in the XMT low energy scan. Figure 6b shows this plot, where a global threshold can be set so that the level of misclassification leads to the grade indicated by the SEM/EDS analysis. It should be noted that this figure is plotted on the basis of the cumulative $2 \mathrm{D}$ area of the grains rather than the number of grains.

a)

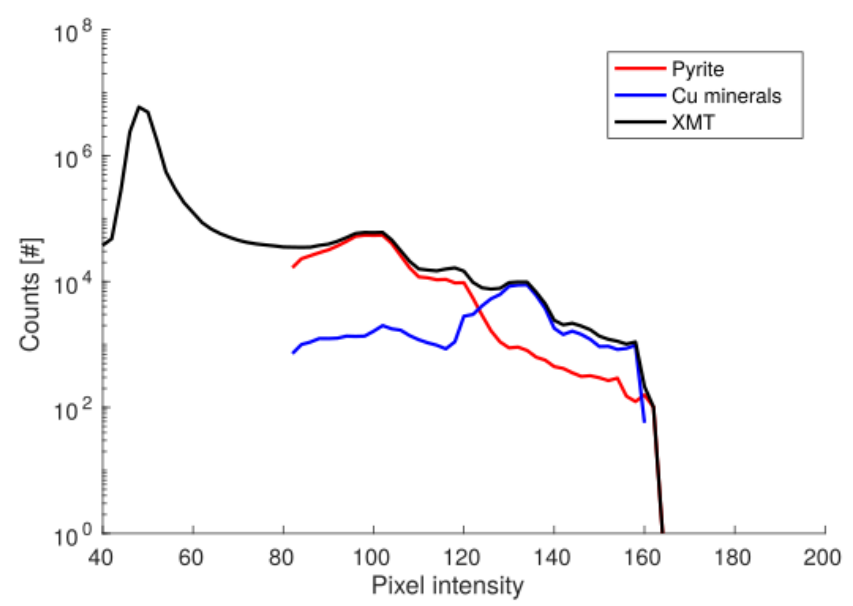

b)

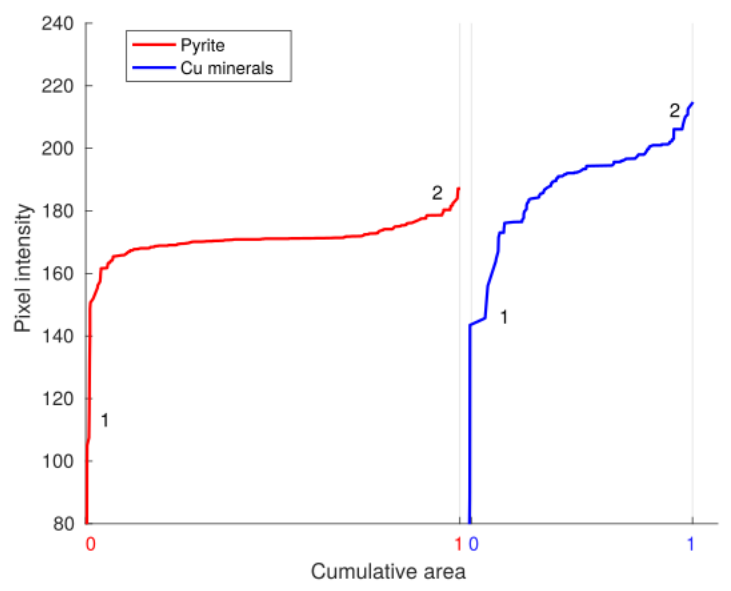

Figure 6 Tracked grains pixel intensity by mineralogy. a) Image intensity histogram showing the XMT pixel value along with the respective histograms of pyrite and copper minerals grains for a particular fragment. b) Pyrite and copper ores grains from all scanned fragments have been sorted by their pixel intensity so a global threshold can be set in order to achieve same composition given by the SEM analyser. Label ' 1 ' shows the result of boundary/partial volume effects, which lower the grains' pixel intensity. Beam hardening causes variability in the grains' intensity with depth into the sample (label ' 2 ')

The numbers in Figure $6 \mathrm{~b}$ indicate situations were image artefacts affect the observed pixel intensity, and thus potentially influence the accuracy of the segmentation. Label ' 1 ' shows grains that have an average intensity that is lower, sometimes significantly, than the typical intensity of grains of that type. This is typically the result of the partial volume effect, where pixels at the graingangue boundary have an intensity lower than those in the interior of the grain. This artefact is critical in smaller grains where the boundary pixels constitute a large proportion of the total. This is why even though there are quite a large number of grains in this tails of the distributions, they do not contribute much to the total area. Beam hardening is an acquisition artefact that makes the measured intensity dependent on the position of the grain within the sample. This artefact is 
responsible for some of the subtler variability in the intensity of the grains within the same type (label ' 2 ') and, unlike partial volume effects, its influence is not restricted to small grains, though its effect can be reduced using a combination of filters and appropriate reconstruction techniques (Hsieh et al., 2000). For the copper minerals, another source of variability is that although chalcopyrite is the dominant mineral in this class, it is not the only one, with different copper minerals having different attenuations.

After obtaining the desired threshold from Figure 6 (an intensity of approximately 175), this value can be applied to all XMT volume images so that simplified 3D mineral maps can be obtained in which gangue, pyrite and copper minerals are identified. This curve also allows for the evaluation of the level of uncertainty and error in the classification of mineral phases as one can calculate the cumulative area that will be misclassified by a given intensity threshold. For the chosen value of 175 , pyrite grains accounting for less than $10 \%$ of the total area (and thus volume when extended into 3D) are wrongly assign as copper grains

\section{Results and discussion}

After polishing and imaging in the SEM scanner all 37 fragments were successfully registered to their corresponding 3D-XMT image. Grain size quantification and mineralogy classification results are presented in this section.

\subsection{Grain size distribution}

After applying the tracking algorithm, only grains that appear in both modalities are considered. This removes the bias created by the higher resolution achieved by SEM in comparison to XMT. For these grains, the grain size distribution by number is show in Figure 7. Grain's equivalent diameter is hereafter considered as proxy for the actual mineral size and all size distributions are calculated by number. XMT information is only obtained here from the virtual 2D slice and so no stereological effect is being assessed. 


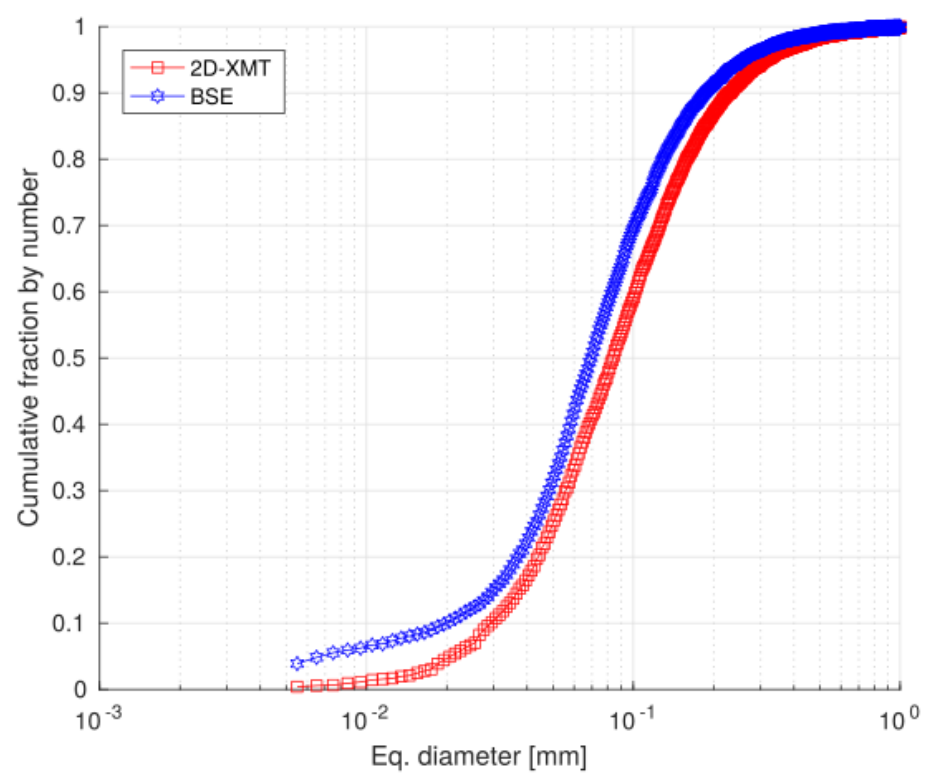

Figure 7 Grain size distribution of SEM slices and registered XMT slice after grain tracking. By considering only tracked grains, the effect of SEM detecting finer grains is removed, as only grains appearing in both modalities are considered.

It can be seen that in comparison to the 2D-XMT registered slice, SEM shows a grain size distribution with more fines. As the linear XMT resolution is almost 8 times (2.25 $\mu \mathrm{m}$ compared to $16.1 \mu \mathrm{m})$ lower than in the SEM images, different effects start to have relevance. Following what Lin et al. (2015) proposed for quantifying error in grain size and considering SEM as the true value for each grain size, we can study the error in XMT volume quantification. Figure 8a shows for each grain their size (equivalent circular diameter) given by the $2 \mathrm{D}$ registered slice in $\mathrm{XMT}$ and their size given by SEM. As expected, the error decreases with grain size. A linear fit to the data shows an intercept of $2 \mu \mathrm{m}$ and a slope of $0.79\left(R^{2}=0.92\right)$, indicating the goodness of the fit. The intercept can be explained by the SEM resolution and a slope less than 1 indicates that XMT introduces a bias that makes grains appear to be bigger than they actually are. This mismatch can be adjusted by changing the threshold used to segment the sulphide grains from the non-sulphide gangue, though this would require adjustments away from that obtained using the Otsu algorithm. A similar algorithm could be applied to that proposed in the paper of Lin et al. (2015), but with the SEM based grains as the reference size.

Confidence limits of $95 \%$ can be used to estimate the precision of XMT when quantifying grain size. Figure $8 \mathrm{~b}$ plots the standard deviation of the relative error as function of grain size, showing that, for this particular case, grains larger than $0.5 \mathrm{~mm}$ in diameter present a random error (uncertainty) of less than $10 \%$ in their quantified size at the given XMT resolution.

a)

b) 

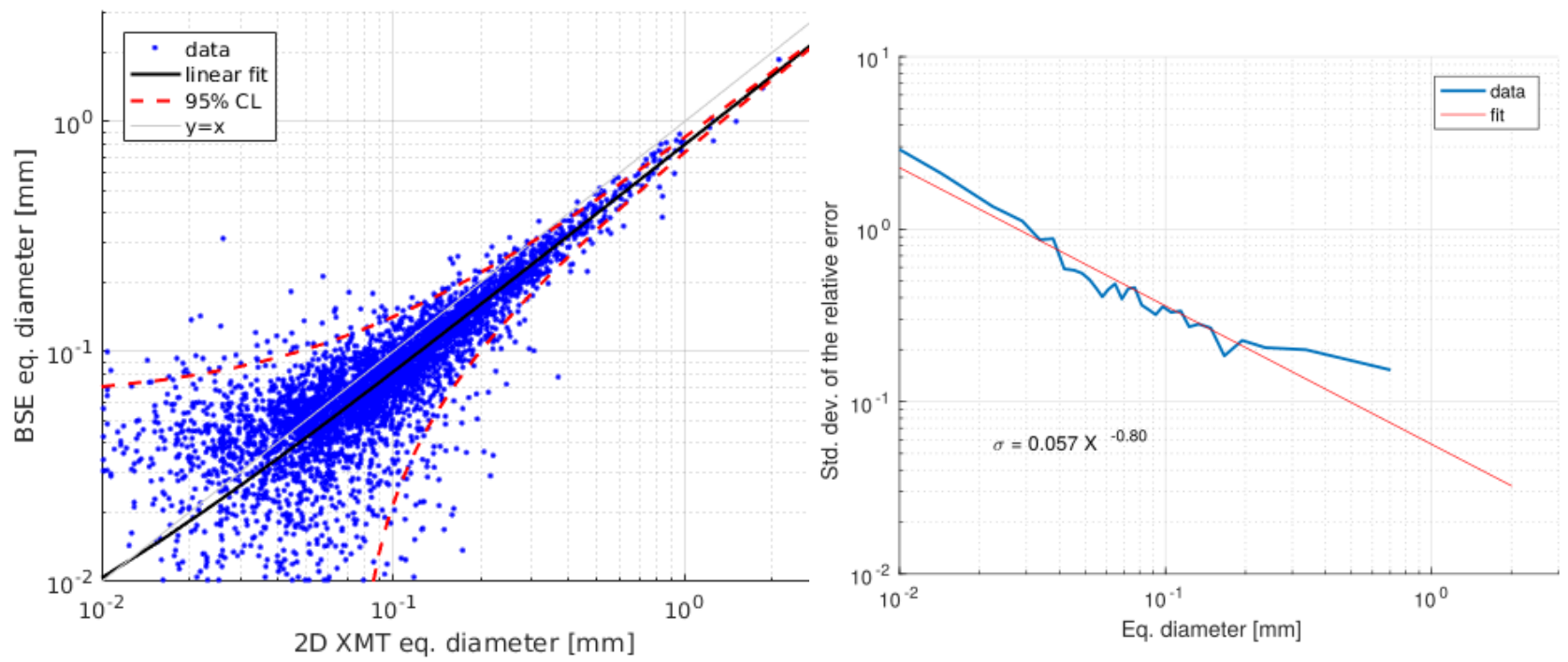

Figure 8 Assessing XMT error in grain size. After grain tracking, SEM grain segmentation can be used as a standard to assess XMT precision on measuring grain size. a) linear model fits the data with an $R^{2}$ of 0.918 , a slope of 0.79 and an intercept of $2[\mu \mathrm{m}]$. b) standard deviation of the relative error between SEM and XMT

3D information of the tracked grains is also available and can be quantified as well. Table 3 shows the $P_{20}$ and $P_{80}$ obtained from the SEM assessment, the 2D registered XMT slice and the 3D XMT volume. Here the effect dominating the difference between the 2D and 3D data is the stereological effect. This effect makes the 2D grain size distribution, obtained from the slices, appear finer than the actual 3D size distribution as the slices will occur through random locations on the grains and not just at their full diameters.

Table 3. Grain size information obtained from the tracked grains. After tracking the higher resolution from SEM is corrected by only considering grains detected by both devices. The observed difference is mainly due to stereological effects.

\begin{tabular}{lccc}
\hline & SEM & 2D-XMT & 3D-XMT \\
\hline $\mathrm{P}_{20}[\mu \mathrm{m}]$ & 36.8 & 44.0 & 66.4 \\
$\mathrm{P}_{80}[\mu \mathrm{m}]$ & 129.0 & 158.2 & 234.3 \\
$\operatorname{Res}[\mu \mathrm{m}]$ & 2.25 & 16.1 & 16.1
\end{tabular}

This effect has been studied before by performing the XMT imaging after the polishing of the fragments and without a grain tracking correction (Gay et al., 2006). This meant that only the average behaviour of the grains in the rest of the fragment and polished section were compared, rather than involving a comparison of exactly the same grains. In this study, XMT contains all the grain size information as polishing was done after scanning. Figure 9 shows the grain size distribution for the 2D-XMT and 3D-XMT versions of the tracked grains (left) and the cumulative distribution function of the stereological effect over the mineral grains. In addition, the stereological effect for 
a number of other shapes are included for comparison (right; more shapes in Sahagian \& Proussevitch, 1998). It can be seen that for the mineral grains, $90 \%$ appear smaller than they actually are and that their distribution differs significantly from that of the other shapes commonly used for these corrections. Also, size overestimation has a long tail, with grains appearing up to 3 times their actual size. It is worth noting that, by using only XMT data (the 3D data and the virtual 2D slice through the data), the effect of higher resolution in SEM images, as well as the uncertainty in the grain size (Figure 8), is avoided and only the stereological effect remains as the source of difference. 
a)

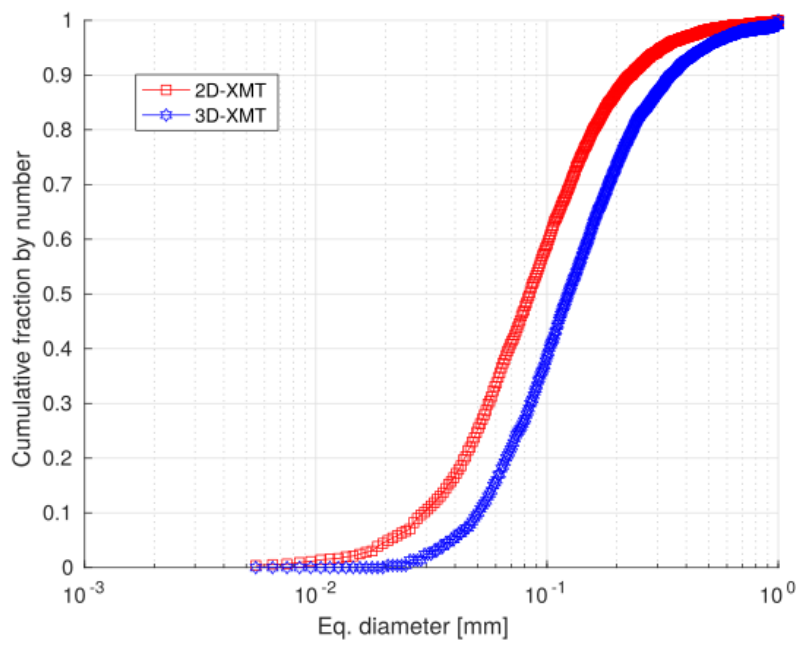

b)

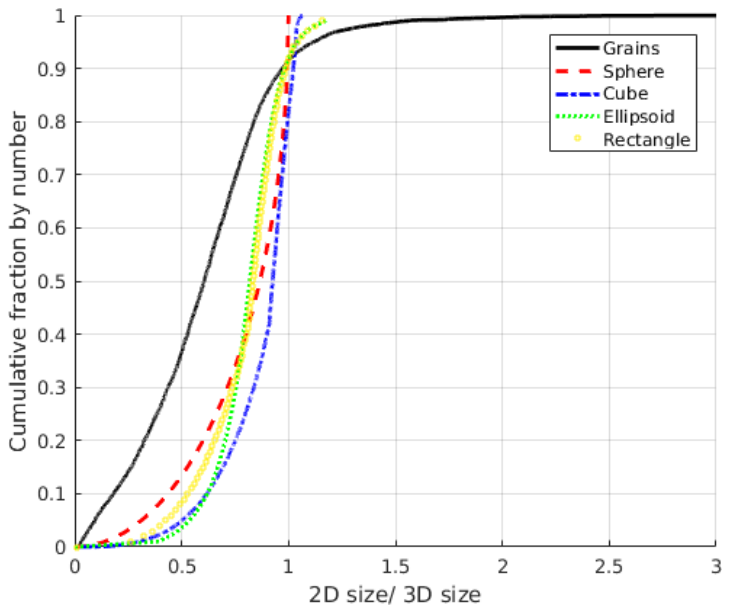

Figure 9 Stereological effect. Using the XMT 3D information of grain sizes, one can obtain the stereological effect of the 2D information given by the SEM slices. a) shows the grains size distribution of the 2D and 3D images, b) shows the cumulative probability distribution function of the error in size for the mineral grains and some convex shapes.

This information gives useful insight into the effect created by studying grain sizes based only on 2D images, and provides the necessary data for an ore-specific stereological correction for further studies with the same ore. Moreover, while a physical slice is required to perform the SEM-based calibration, virtual 2D slices can be traced over the 3D data from XMT to provide a statistically robust stereological correction.

\subsection{Simplified 3D mineralogical map}

As only a simplified mineral map will be obtained, copper minerals, pyrite, gangue and porosity are the phases to be identify. Porosity and gangue are easily segmented from the bright phase, but in the latter, no obvious threshold can be found directly from the intensity histogram (see Figure 6a). Using the procedure described in Section 2.2, a global threshold that creates the same grade specified by the SEM modal mineralogy information was found. This threshold produces a misclassification, due to X-ray artefacts discussed in Section 2.4. This error can be quantified according to the grain size, as shown in Table 5, by grouping grains in size categories and evaluating the percentage of false positives (pyrite classified as copper) and false negatives (copper classified as pyrite). As expected, the miss-classification decreases with grain size, especially for the false negatives. This is because false negatives are due to partial volume effects, which are strongly correlated with grain size. Conversely, beam hardening effects grains of all sizes and is the dominant reason for the false positives. 
Table 4. Mineral misclassification by size.

\begin{tabular}{ccc}
\hline Size range $[\mu \mathrm{m}]$ & $\begin{array}{c}\text { False } \\
\text { positives } \\
\text { (\% of grains) }\end{array}$ & $\begin{array}{c}\text { False } \\
\text { negatives } \\
\text { (\% of grains) }\end{array}$ \\
\hline$<25.3$ & 7.62 & 7.85 \\
$25.3-53.1$ & 10.32 & 7.94 \\
$53.1-82.5$ & 6.90 & 0.91 \\
$82.5-124$ & 9.33 & 2.3 \\
$>124$ & 10.42 & 0.0
\end{tabular}

Figure 10 and 11 show two typical fragments as examples; grey represents gangue, brown pyrite and yellow the copper mineral grains. Porosity is rendered transparent.

a)

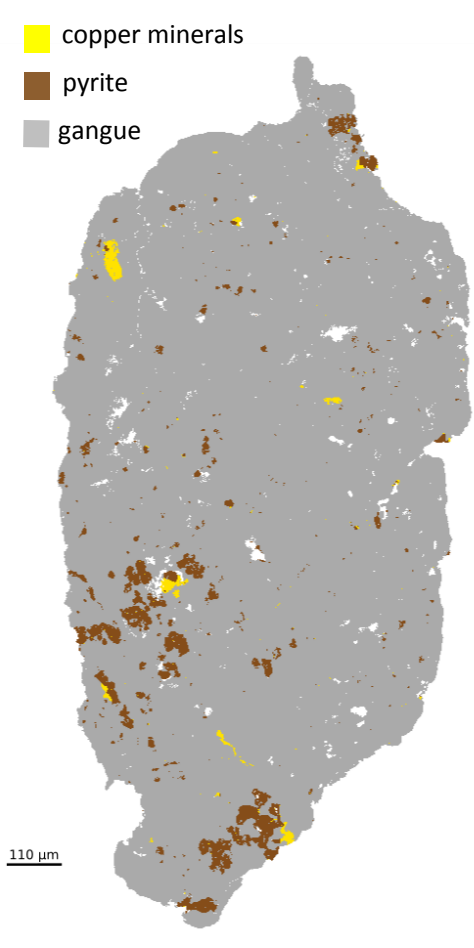

b)

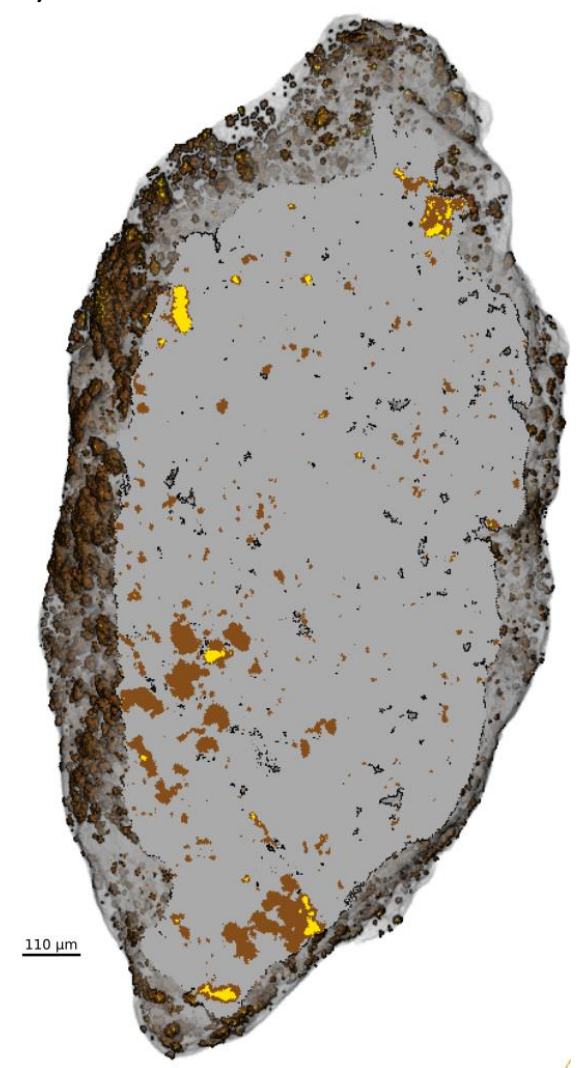

c)

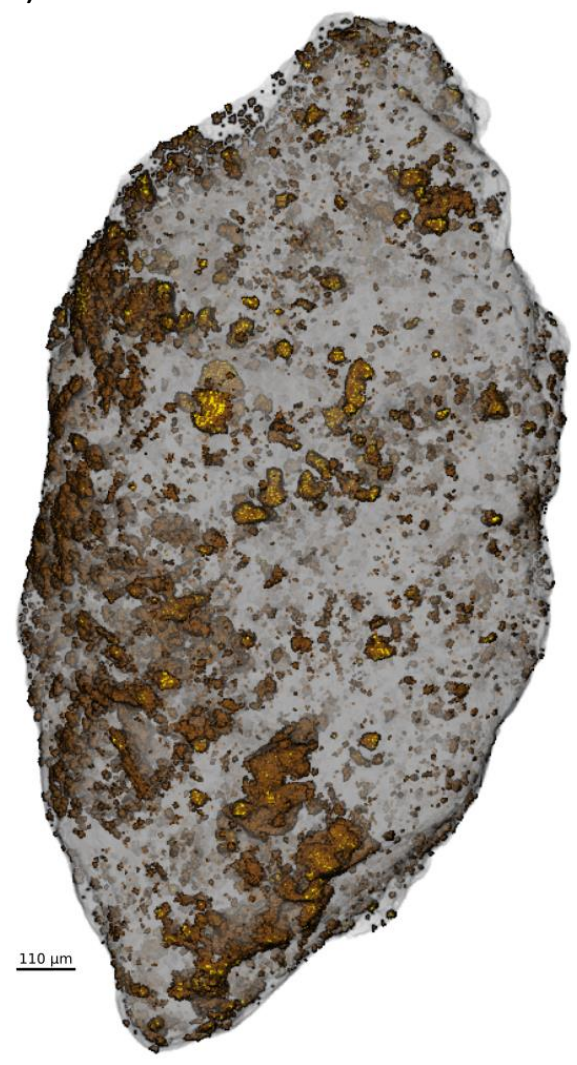

Figure 10 Resulting calibrated 3D mineral map. Applying the threshold found by Figure 6, a 3D mineral map distinguishing between gangue, porosity, pyrite and copper minerals is obtained. a) EDS based classification, b) same slice using the threshold found and c) the resulting 3D mineral map 
a)

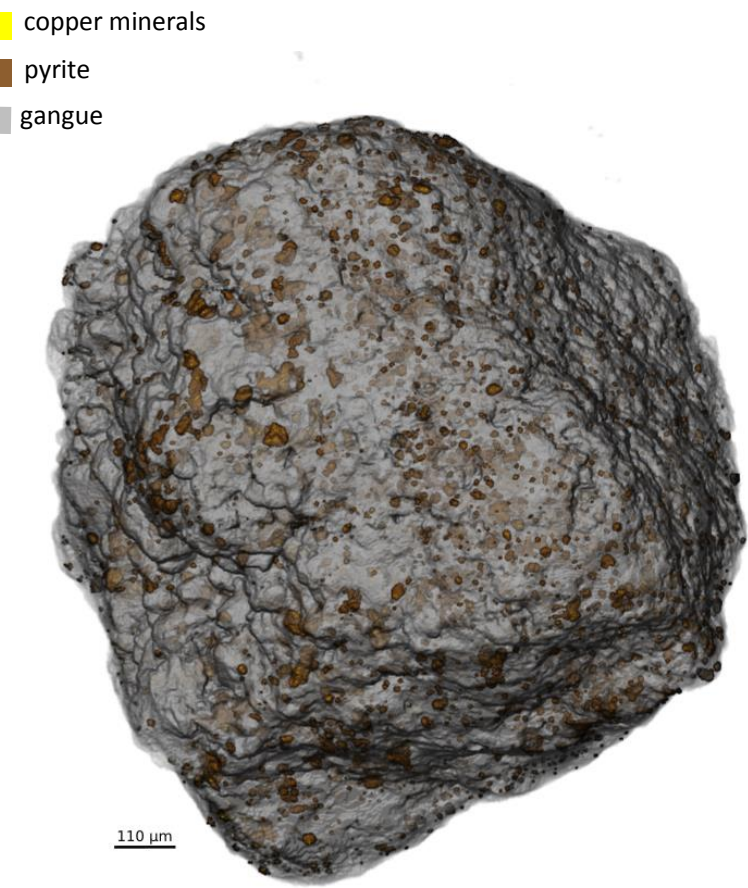

b)

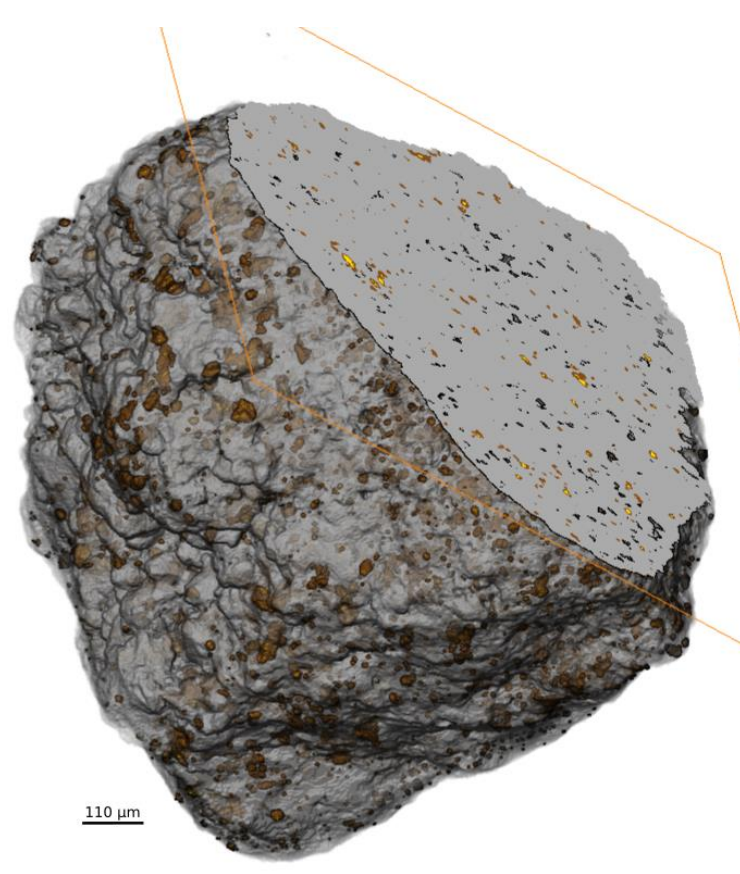

Figure 11 Resulting calibrated 3D mineral map and slice. Another example of the 3D mineral map obtained from SEM/EDS calibrated low energy XMT scans. a) rendered volume and b) virtual slice

The information provided by this 3D mineral map adds a new dimension to all previous analyses done using XMT images alone. Now mineralogy can be associated with mineral liberation, grain size, grain shape, fracture, crack network creation, etc., all in 3D. Limitations of the methodology, however, are the result of limitations of both analytical devices. Critical ones are, having a large enough sample size in order to achieve statistically significant results, the resolution achieved by the XMT scan (heavily dependent on the particles size) and more minor consideration being possible mineral misclassification by the SEM/MLA scanner (Lee et al., 2011). Also, since only single energy level radiography is used, materials with similar attenuation coefficients will not be distinguishable from one another. For those materials, multiple energy radiography will be needed (Lin et al., 2013; Long et al., 2009). Also, the methodology is bounded to the mineralogy presented by the SEM analysis so it can't distinguish minerals that have not been identified in the SEM analysis.

The combination of the XMT and SEM images does alleviate some of the sample size issues associated with using the latter method alone, especially with respect to grain scale information. This is because a slice through a fragment will only intersect a small fraction of the grains present, especially in systems such as the one examined in this paper where the grains are much smaller than the ore particles. By calibrating the XMT images, a far larger sample size of grains is available and thus less slicing of fragments is required. For instance, Table 5 shows the fractions of gangue, pyrite 
and copper minerals for the particles as obtained from the SEM analysis and compares them to the results obtained from the full volume using the calibrated XMT results.

Table 5. Mineralogy obtained from SEM and XMT. Proportion, by area or volume, of gangue, pyrite and copper minerals in the fragments scanned with low energy XMT. Differences show the statistical biased of SEM for a small sample size can be compensated with the 3D information given by XMT

\begin{tabular}{lccc}
\hline & SEM \% & 2D-XMT \% & 3D-XMT \% \\
\hline Gangue & 95.47 & 95.69 & 96.74 \\
$\begin{array}{l}\text { Pyrite } \\
\text { Copper }\end{array}$ & 3.07 & 3.17 & 2.49 \\
minerals & 1.46 & 1.14 & 0.77
\end{tabular}

This shows that while the limited sample size exposed for the SEM analysis is useful for calibration and can provide an analysis for a far wider range of minerals, it can be misleading in terms of the actual particle scale variability, showing in this example an overrepresentation of the presence of sulphide minerals (pyrite and copper minerals). This does not mean that XMT inherently provides a better analysis than MLA/SEM, but rather that by combining the two methods, the fragment scale compositional uncertainty associated with taking only a single slice through the fragment can be eliminated, which means that the combined methodology will inherently require the analysis of fewer fragments to achieve a statistically valid result than either of the methods on their own, due to the minimisation of the uncertainties associated with mineral misclassification in the XMT images and the limited number of grains intersected in a 2D section for the MLA/SEM. The authors acknowledge that for a representative mineralogical study more than 34 sample need to be assessed to fully represent the variability of the ore under study, though the purpose of this paper was to present a methodology and to study mineral grain scale effects, for which there is a statistically valid sample size.

\section{Conclusions}

A methodology for combining XMT and SEM images so that more detailed mineral information can be obtained from the 3D XMT images has been presented. The methodology makes use of a tracking algorithm that matches both imaging modalities at the grain level. Tested in copper sulphide ores, the methodology allows the acquisition of a 3D segmented images of ore fragments where copper minerals, pyrite, gangue and porosity are differentiated. Segmentation thresholds are calibrated using the SEM automatic mineral classification, whilst the XMT grain size quantification error is also evaluated. SEM 2D image-based grain size distributions can also be corrected for stereological 
effects using the 3D information on the grains in the XMT volume. The proposed methodology differs from previous work with XMT and SEM in the way in which data from both devices is combined so that information from one can be transferred to the other at the level of the individual grains rather than at an averaged macroscopic level. This novel approach allows the confidence limits and uncertainty in both the mineral classification and grain scale quantification to be assessed. Possible limitations of the methodology are the presence of minerals with similar X-ray attenuation coefficients, making them harder to be segmented only using a low energy scan. In this case study it was possible to distinguish the pyrite from the copper sulphide species, but it was impossible to distinguish the different copper minerals from one another due to a combination of similar X-ray attenuations and the small number of grains of the minor copper species intercepted in the 2D sections. For cases like these, a dual energy, or even multi-energy, radiography technique together with a larger number of sections might be necessary. The use of a combination of multiple energy XMT scans and SEM information is thus the logical progression of this work.

\section{Acknowledgements}

This study was performed in the Rio Tinto Centre for Advanced Mineral Recovery at Imperial College London. XMT scans were performed at the Manchester X-ray Imaging Facility at Harwell, which was funded in part by the EPSRC platform grant (EP/I02249X/1). The underlying raw data is not shared online due to its huge size, but representative sample data is included in the figures. The authors gratefully acknowledge Rio Tinto for their financial support for this project.

\section{References}

[1]. Berger, M. J., Hubbell, J. H., Seltzer, S. M., Chang, J., Coursey, J. S., Sukumar, R., Zucker, D. S., Olsen, 2010. XCOM: Photon Cross Sections Database. NIST Standard Reference Database 8 (XGAM).

[2]. Charikinya, E., Bradshaw, S., \& Becker, M. (2015). Characterising and quantifying microwave induced damage in coarse sphalerite ore particles. Minerals Engineering, 82, 14-24.

[3]. Cnudde, V., \& Boone, M. N. (2013). High-resolution X-ray computed tomography in geosciences: A review of the current technology and applications. Earth-Science Reviews, 123, 117.

[4]. Dhawan, N., Safarzadeh, M. S., Miller, J. D., Moats, M. S., Rajamani, R. K., \& Lin, C. L. (2012). Recent advances in the application of X-ray computed tomography in the analysis of heap leaching systems. Minerals Engineering, 35(0), 75-86.

[5]. Fandrich, R., Gu, Y., Burrows, D., \& Moeller, K. (2007). Modern SEM-based mineral liberation analysis. International Journal of Mineral Processing, 84(1-4), 310-320.

[6]. Garcia, D., Lin, C. L., \& Miller, J. D. (2009). Quantitative analysis of grain boundary fracture in the breakage of single multiphase particles using X-ray microtomography procedures. Minerals 
Engineering, 22(3), 236-243.

[7]. Gay, S. L., Morrison, R. D., \& Kruttschnitt, J. (2006). Using two dimensional sectional distributions to infer three dimensional volumetric distributions - Validation using tomography. Particle and Particle Systems Characterization, 23(3-4), 246-253.

[8]. Ghorbani, Y., Becker, M., Petersen, J., Morar, S. H., Mainza, A., \& Franzidis, J. P. (2011). Use of $\mathrm{X}$-ray computed tomography to investigate crack distribution and mineral dissemination in sphalerite ore particles. Minerals Engineering, 24(12), 1249-1257.

[9]. Hsieh, J., Molthen, R. C., Dawson, C. a, \& Johnson, R. H. (2000). An iterative approach to the beam hardening correction in cone beam CT. Medical Physics, 27(1), 23-29.

[10]. Jordens, A., Marion, C., Grammatikopoulos, T., \& Waters, K. E. (2016). Understanding the effect of mineralogy on muscovite flotation using QEMSCAN. International Journal of Mineral Processing.

[11]. Kapur, J.N., Sahoo, P.K., Wong, A.K.C., 1985. A New Method for Gray-level Picture Thresholding Using the Entropy of the Histogram. Computer Vision, Graphics, and Image Processing 29(3), pp. 273-285.

[12]. Kyle, J. R., \& Ketcham, R. A. (2015). Application of high resolution X-ray computed tomography to mineral deposit origin, evaluation, and processing. Ore Geology Reviews, 65, 821839.

[13]. Lee, J., Acar, S., Doerr, D. L., \& Brierley, J. A. (2011). Comparative bioleaching and mineralogy of composited sulfide ores containing enargite, covellite and chalcocite by mesophilic and thermophilic microorganisms. Hydrometallurgy, 105(3-4), 213-221.

[14]. Leißner, T., Hoang, D. H., Rudolph, M., Heinig, T., Bachmann, K., Gutzmer, J., Peuker, U. A. (2016). A mineral liberation study of grain boundary fracture based on measurements of the surface exposure after milling. International Journal of Mineral Processing. In Pres, http://dx.doi.org/10.1016/j.minpro.2016.08.014

[15]. Lin, C. L., Hsieh, C.-H., Tserendagva, T.-A., \& Miller, J. D. (2013). Dual energy rapid scan radiography for geometallurgy evaluation and isolation of trace mineral particles. Minerals Engineering, 40, 30-37.

[16]. Lin, Q., Neethling, S. J., Dobson, K. J., Courtois, L., \& Lee, P. D. (2015). Quantifying and minimising systematic and random errors in X-ray micro-tomography based volume measurements. Computers \& Geosciences, 77, 1-7.

[17]. Lin, Q., Barker, D. J., Dobson, K. J., Lee, P. D., \& Neethling, S. J. (2016a). Modelling particle scale leach kinetics based on X-ray computed micro-tomography images. Hydrometallurgy, 162, 2536.

[18]. Lin, Q., Neethling, S. J., Courtois, L., Dobson, K. J., \& Lee, P. D. (2016b). Multi-scale quantification of leaching performance using X-ray tomography. Hydrometallurgy, 164, 265-77

[19]. Long, H., Swennen, R., Foubert, A., Dierick, M., \& Jacobs, P. (2009). 3D quantification of mineral components and porosity distribution in Westphalian $\mathrm{C}$ sandstone by microfocus X-ray computed tomography. Sedimentary Geology, 220(1-2), 116-125.

[20]. Miller, J. D., Lin, C. L., Garcia, C., \& Arias, H. (2003). Ultimate recovery in heap leaching operations as established from mineral exposure analysis by X-ray microtomography. International Journal of Mineral Processing, 72(1-4), 331-340.

[21]. Naderi, H., Abdollahy, M., Mostoufi, N., Koleini, M. J., Shojaosadati, S. A., \& Manafi, Z. (2011). 
Kinetics of chemical leaching of chalcopyrite from low grade copper ore: Behavior of different size fractions. International Journal of Minerals, Metallurgy and Materials, 18(6), 638-645.

[22]. Otsu, N. 1979. A threshold selection method from gray-level histograms. IEEE Transactions on Systems, Man, and Cybernetics, 9, 62-66.

[23]. Sahagian, D. L., \& Proussevitch, A. A. (1998). 3D particle size distributions from 2D observations: Stereology for natural applications. Journal of Volcanology and Geothermal Research, 84(3-4), 173-196.

[24]. Sok, R. M., Varslot, T., Ghous, A., Latham, S., Sheppard, A. P., \& Knackstedt, M. a. (2010). Pore Scale Characterization of Carbonates at Multiple Scales: Integration of Micro-CT, BSEM, and FIBSEM. Petrophysics, 51(6), 379-387.

[25]. Sutherland, D. N., \& Gottlieb, P. (1991). Application of automated quantitative mineralogy in mineral processing. Minerals Engineering, 4(7-11), 753-762.

[26]. Tungpalan, K., Wightman, E., \& Manlapig, E. (2015). Relating mineralogical and textural characteristics to flotation behaviour. Minerals Engineering, 82, 136-140.

[27]. Van Geet, M., Swennen, R., \& David, P. (2001). Quantitative coal characterisation by means of microfocus X-ray computer tomography, colour image analysis and back-scattered scanning electron microscopy. International Journal of Coal Geology, 46(1), 11-25. 